\title{
Prediction of Propagation Path Loss for Stratospheric Platforms Mobile Communications in Urban Site LOS/NLOS Environment
}

\author{
Iskandar and Shigeru Shimamoto \\ Graduate School of Global Information and Telecommunication Studies, Waseda University \\ 1011 Okuboyama Nishi-Tomida Honjo-shi Saitama 367-0035, Japan \\ Email: iskandar@fuji.waseda.jp, shima $a$ waseda.jp
}

\begin{abstract}
Recently, there are of great interests on employing stratospheric platform as a new means of providing IMT-2000. One important issue that has not been much investigated is the propagation characteristic of its link. This paper aims at evaluating the impact of elevation and azimuth angles variation to the propagation mechanism in urban environment for mobile communication link. In order to examine the propagation loss experienced by this system in urban area, we developed the building block model and the ray tracing tool for simulation. We then apply the ray tracing tool with respect to the angle variations. As a result, propagation loss as a function of elevation and azimuth angles is obtained. We compared the result with a physical-statistical model for verification. Finally, link budget analysis and estimation to the required transmitted power at several transmission rates for the application of IMT-2000 is observed. Different scenario, which means different azimuth angles clearly show the critical limitations for mobile communication using a concept of stratospheric platform.
\end{abstract}

\section{INTRODUCTION}

Currently we have two well established methods for providing wireless communication services. Those are the traditional satellite and the terrestrial systems. In recent years, a novel wireless concept that has been attracting much the attention is proposed. It is based on aerial vehicles operating in the stratosphere located at around $17-22 \mathrm{~km}$ above the ground known as stratospheric platform (SPF) or high altitude platform station (HAPS) [1]-[3]. With this concept, we are able to exploit much the advantages and at the same time overcome the shortage of both the traditional systems. The most important advantages of employing SPF are high elevation angles, broad coverage, low propagation delay, low-cost operation, easy and incremental deployment, and ability to move around in emergency situation [1]. However, immature airship technology, stabilization, and onboard antenna technology are also challenging [4]. SPF is expected to avoid some limitations of the traditional systems. Those are in the sense of a huge number of base stations required by the terrestrial system, limitation of the minimum cell size on the ground involved in GEO satellite system, and suffer from handover problem faced by LEO/MEO satellite system. With these great advantages of SPF, the ITU has allocated the spectrum to this system at $2 \mathrm{GHz}$ for $3 \mathrm{G}$ mobile systems [5], 48/47 GHz for the usage worldwide [6], and 31/28 $\mathrm{GHz}$ for certain Asian countries [7].
As the designing and developing of the SPF is now being in progress, the potential applications have been proposed and studied. Some of them are broadband fixed access, digital broadcasting, remote sensing and earth observation, and the next generation of mobile communication. Frequency reuse is definitely required in order such a system to deliver high spectral efficiency by a large number of spot beams. A method of improving the sys-tem capacity of broadband services has been evaluated in [8]. The cellular structure on the ground was proposed to be the solution of an application of frequency reuse [9]. An efficient cellular structure by means of an onboard of antenna array is also examined in [10]. By making use of frequency reuse, SPF system will suffer from the main problem of interference that can reduce the system capacity. Some of the studies related to interference experienced by SPF system have been evaluated in [11]. Joint system between SPF and terrestrial system has also been examined [12] as well as integration of SPF within UMTS terrestrial network [13].

The detail propagation path loss model for the case of SPF is essential in order to improve the results of the aforementioned studies. Although line of sight (LOS) situation is expected to be the most predominant link in SPF communication, however non-line of sight (NLOS) situation should also be evaluated. Therefore, both LOS and NLOS propagation model is required to be investigated. In this paper, we examined the propagation path loss model for mobile communication employing the SPF by taking an example in urban environment using ray tracing algorithm. Our previous work has investigated the performance of SPF communication in comparison with LEO satellite system. In this study fade and non-fade duration derived from measurement is incorporated to our simulation environment [14]. We have also studied channel characterization of the SPF link and evaluated its performance [15]. The channel parameters that were obtained from measurement are then used to evaluate the SPF channel performance. However, an experiment has been carried out only in a semi-urban environment. Propagation channel model in the city for the case of SPF system in which the mobile station (MS) mostly exists almost has not been reported. Therefore, this paper proposed the propagation model that examines the impact of elevation angles variation against the propagation mechanism in urban environment for mobile communication link served by the SPF system. 


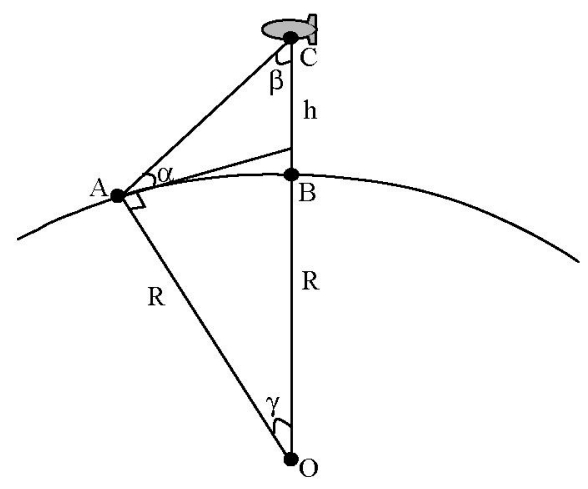

Fig. 1 Geometrical parameters of stratospheric platform

\section{SpF System Geometry}

The geometry of SPF system is described in Fig. 1. SPF is positioned at an altitude $h$ (point $\mathrm{C}$ ) with the sub-platform point (the point vertically below the intended platform location) is in point B. In Fig. 1, point A denotes the position of a user served by the SPF having elevation angle $\alpha$. Point $\mathrm{O}$ represents the earth center and $R$ is the earth radius. From the principle of trigonometry we can express

$$
\begin{gathered}
\frac{O A}{\sin \beta}=\frac{O C}{\sin (90+\alpha)}=\frac{O C}{\cos \alpha}, \\
\sin \beta=\frac{R}{R+h} \cos \alpha .
\end{gathered}
$$

Assuming the earth surface is perfect sphere, the arc $A B$ indicates the radius $(d)$ of SPF coverage on the ground and might be expressed through the following equation

$$
A B=R \gamma .
$$

Let consider the triangle $\mathrm{OAC}$, the total angle of the triangle is $180^{\circ}$ so that angle $\gamma$ can satisfy this equation

$$
\gamma=90^{\circ}-\beta-\alpha
$$

With substitutions of (2)-(4), we are able to rewrite the radius of the SPF coverage in (5) and it is shown graphically in Fig. 2.

$$
d=R\left\{\cos ^{-1}\left(\frac{R}{R+h} \cos \alpha\right)-\alpha\right\} .
$$

According to the above geometrical analysis, the SPF at $20 \mathrm{~km}$ altitude has the capability to cover an area on the ground up to $504 \mathrm{~km}$ in radius for $0^{\circ}$ of elevation angle.

\section{GEographical Data SuRvey}

In this section, we present in part of the site survey result of the building height and visibility. Extensive series of survey were conducted inside three areas of the Tokyo Prefecture (i.e. Shinjuku, Shibuya, and Asakusa) and inside Kiryu area of the Gunma Prefecture. The cumulative probability of building height distribution among those areas is obtained and demonstrated in Fig. 3. We observed that Shinjuku represents as an area in which the probability of building height distribution is

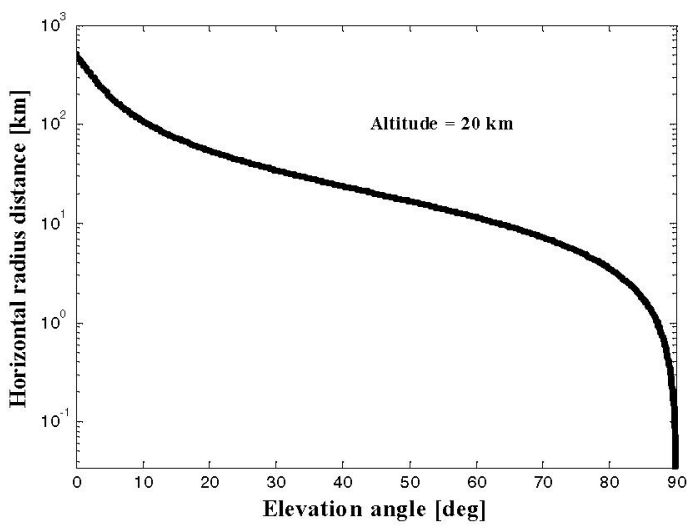

Fig. 2 Radius of SPF coverage (vertical axis on the logarithmic scale)

highest compared with the other areas. From surveys we also observed an average of building height as well as building density [pieces $/ \mathrm{km}^{2}$ ] and the result is depicted in Table 1 .

TABLE I Average BuILding HeIGHT AND DENSITY

\begin{tabular}{|l|c|c|}
\hline Area & $\begin{array}{c}\text { Average building } \\
\text { height }[\mathrm{m}]\end{array}$ & $\begin{array}{c}\text { Building density } \\
\left.\text { [pieces } / \mathrm{km}^{2}\right]\end{array}$ \\
\hline Shinjuku & 25.5 & 290 \\
\hline Shibuya & 25.0 & 200 \\
\hline Asakusa & 22.1 & 580 \\
\hline Kiryu & 19.1 & 7 \\
\hline
\end{tabular}

During the field tests we also examined the visibility in a step of $10^{\circ}$ intervals of elevation angle. The visibility is defined as the ratio of propagation path in LOS situation to the total situation (both LOS and NLOS situation) at each elevation angle from MS's perspective to the SPF. In Fig. 4, the markers (triangles, diamonds, squares, and circles) demonstrate the result of experiments for the visibility of Asakusa area. The lines (solid, dashed, dotted, and dashed-dot) illustrate an approximation of the visibility by equation as a function of elevation $(\alpha)$ and azimuth $(\theta)$ angle through the following expression

$$
p_{v}(\alpha, \theta)= \begin{cases}(\sin \alpha)^{0.01} & ; \theta=0^{\circ} \\ (\sin \alpha)^{\sin \theta} & ; \theta=30^{\circ}, 60^{\circ}, 90^{\circ},\end{cases}
$$

where $\theta=0^{\circ}$ is in the positive $y$-axis direction and $\theta=90^{\circ}$ is in the negative $x$-axis direction explained in Fig. 5. We found that the visibility is a decreasing function against azimuth angle. The same behavior of visibility is also observed for the other areas i.e. for Shinjuku, Shibuya, and Kiryu. However, due to limited space we left the data for evaluation and do not presented in this paper.

\section{DESCRIPTION OF THE MODEL}

\section{A. Stratospheric Platform Ray Tracing Model}

We developed the model for outdoor propagation evaluation for the block of 8 buildings given in Fig. 5 (top view) and Fig. 6 (side view). The model to be presented has been selected to represent the suitable area over which it closes to the characteristic of Asakusa area. The SPF is varied along 


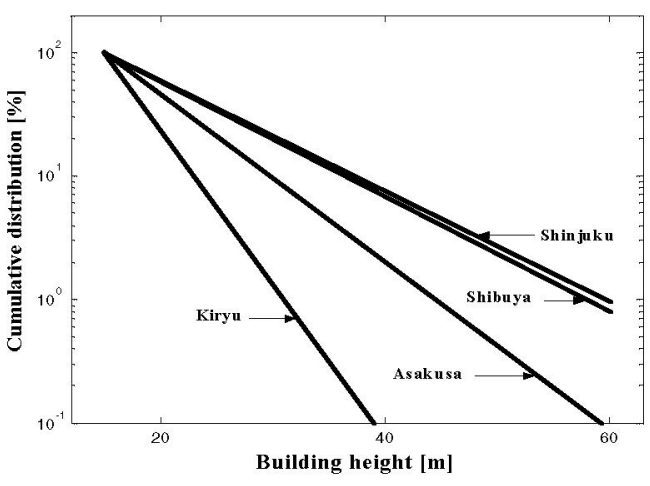

Fig. 3 Building height cumulative distribution

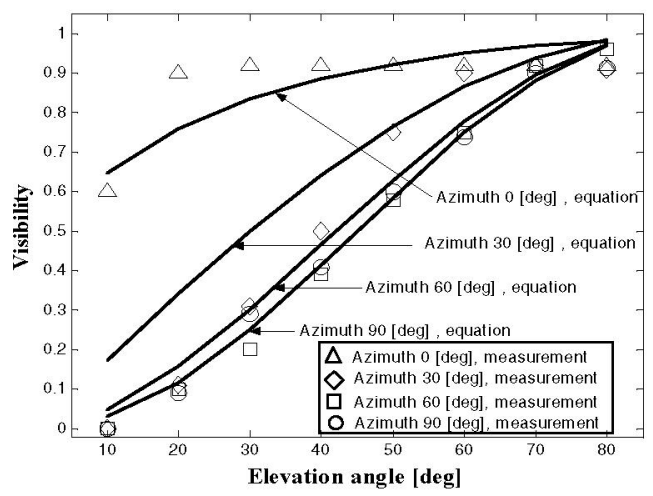

Fig. 4 Visibility for Asakusa area

with elevation angle from $5^{0}$ to $90^{\circ}$ in a step of $1^{0}$. Three different azimuth angles were examined those are $90^{\circ}, 60^{\circ}$, and $45^{\circ}$ Buildings in the model are assumed to have the same height $\left(h_{b}=20 \mathrm{~m}\right)$ and the same width $\left(w_{b}=25 \mathrm{~m}\right)$, but vary in length. All street width $\left(w_{s}\right)$ of $35 \mathrm{~m}$ is assumed to be equal in the model under consideration (slightly wider than real street in Asakusa). The MS has $1.5 \mathrm{~m}$ in height and is located at the center of the street $\left(d_{m}=w_{s} / 2\right)$ between building 1 and building 2 (point A) with the ray impinging the MS from an arbitrary direction. Frequency of $2 \mathrm{GHz}$ has been suggested for the SPF-IMT-2000 services [5] and hence we used this frequency for simulation.

The ray tracing tool we developed follows an approach model proposed by $\mathrm{F}$. Tila [16], but we included diffraction mechanism effects from building rooftops. The mechanism is found to be important for the case of low elevation angle. The model employs a number of virtual transmitters located around the area under test in order to overcome the difficulty of applying the ray launching methods for the large separation such as from the SPF to the MS. The generation of virtual transmitters is repeated when the elevation angle is changed.

We employ nine categories of ray to be included in our simulation. These ray categories are line of sight, single reflection from building, single reflection from street, double reflection from building to street, double reflection from building to building, diffraction from rooftop of building, diffraction from rooftop and single reflection from building, diffraction from rooftop and single reflection from street, and diffraction from rooftop and double reflection from building to street. Rays outside the above categories are assumed to leave the target and terminated from the simulation environment.

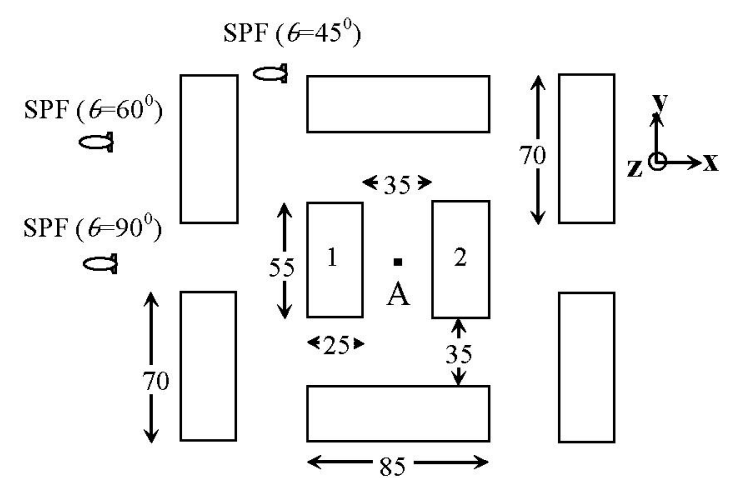

Fig. 5 Top view of the model under test (unit in meter)

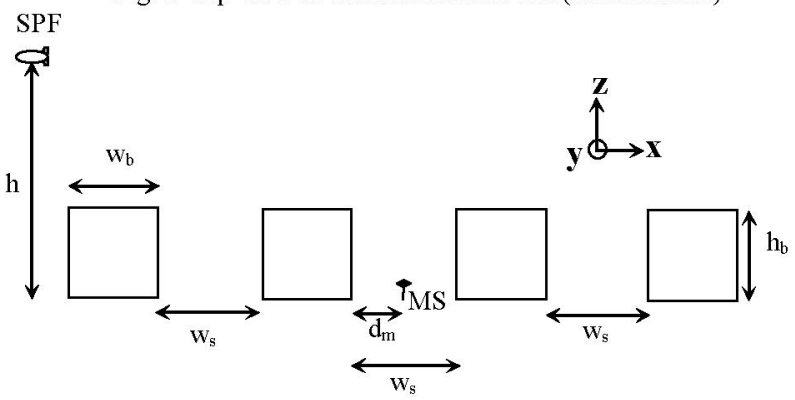

Fig. 6 Side view of the model under test

Diffractions from the building vertical corner are not considered in this work and will be taken into account for future work. Since all surfaces in real propagation environments are finite, also edges and corners have to be considered. When a radio wave encounters an edge of a large object, part of the wave appears to bend into shaded areas behind the edge and this mechanism is called diffraction. It is more important at low frequencies including $2 \mathrm{GHz}$, and hence we included in simulation. Table 2 shows electrical parameter that is used in ray tracing simulation throughout the prediction in this paper. Buildings are assumed to be made of concrete, where $\mathcal{E}_{r}$ and $\sigma$ are dielectric constant and conductivity, respectively. In the prediction, two situations were observed, those are LOS situation and NLOS situation (when the direct ray is obstructed by the building nearby the MS position). As a result, we are able to obtain the power impinging the MS separately from direct LOS ray, multipath scattered power (total power from rays except direct LOS ray), and the total complex vector summation.

TABLE II

Electrical Parameters Used In the Ray tracing Simulation [17]

\begin{tabular}{|c|c|c|}
\hline & $\varepsilon_{r}$ & $\sigma\left[\Omega^{-1} m^{-1}\right]$ \\
\hline Building & 3 & 0.005 \\
\hline Street & 15 & 7 \\
\hline
\end{tabular}

\section{B. Analytical Model}

To compute loss power when the rays intersect with an object we used geometrical optics (GO) and uniform theory of diffraction (UTD). UTD is an extension method of a GO to include diffraction. Both transmitting and receiving antennas are assumed to be an isotropic antenna with a $0 \mathrm{dBi}$ gain. Therefore, an electric field of ray arriving at the receiving 
antenna is calculated using the following formulas for direct ray $\left(E_{L O S}\right)$, reflected ray $\left(E_{R}\right)$, and diffracted ray $\left(E_{D}\right)$, respectively.

$$
\begin{gathered}
E_{L O S}=E_{0} \frac{e^{-j \beta d_{0}}}{d_{0}}, \\
E_{R}=E_{0} \cdot \bar{R} \frac{e^{-j \beta\left(s_{1}+s_{2}\right)}}{s_{1}+s_{2}}, \\
E_{D}=\frac{E_{0}}{s^{\prime}} \cdot \bar{D} \sqrt{\frac{s_{3}}{s\left(s+s_{3}\right)}} e^{-j \beta\left(s+s_{3}\right)},
\end{gathered}
$$

where $\beta=\lambda / 2 \pi$ denotes the propagation constant, $E_{0}$ represents an emitted electric field from transmitter, $d_{0}$ corresponds to the direct path length, $s_{1}$ indicates the distance from the source to the reflection point, $s_{2}$ is the distance from reflection point to the receiver, $s_{3}$ represents the path length from the source to the diffraction point, and $\mathrm{s}$ is the path length from diffraction point to the receiver. $\bar{R}$ represents a Fresnel dyadic reflection coefficient and $\bar{D}$ denotes the dyadic finite conductivity edge diffraction coefficient. These two coefficients follow the general formulation presented on [17]. Finally, respective rays for each ray category were added at the MS and expressed as

$$
E_{i}=\sum_{j=1}^{n} E_{j}
$$

where $E_{j}$ is the received electric field of the $j$ th ray for $i$ th category. We assumed the ray tracing algorithm employed in the paper considers up to 9 ray categories as mentioned above. Although the precision can be improved by additional ray category such as double diffraction in combination with multiple reflections from wall and street, however we have to compensate by computational complexity and therefore it is actually time consuming. In this work, the total electric field contribution consists of vector summation of 9 ray categories expressed by

$$
E_{\text {Tot }}=\sum_{i=1}^{9} E_{i} .
$$

Finally, the total propagation loss $(L)$ is expressed as

$$
L=20 \log \left(\frac{\lambda}{4 \pi} \frac{\left|E_{T o t}\right|}{\left|E_{0}\right|}\right)
$$

From (12), now we can demonstrate the propagation path loss for the above-mentioned model as a function of elevation and azimuth angle and presented in the following section.

\section{Propagation Result and Comparison with Physical- STATISTICAL MODEL}

There is no measurement result for the propagation loss in urban environment for the case of SPF until now. Therefore we compared our ray tracing results with an approach model of the physical-statistical model proposed in [18] for verification. The model allows predictions to be made for systems operated in the areas where direct measurements are unavailable. The model also employs a simple approach and thorough analysis. We apply the model to the same building block as described in

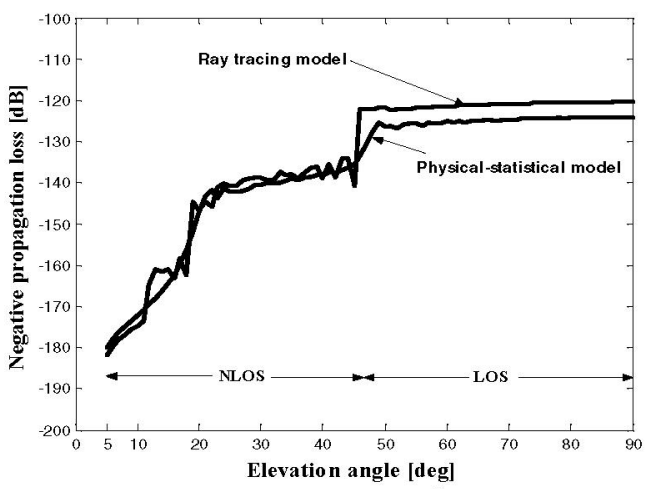

(a) azimuth $90^{\circ}$

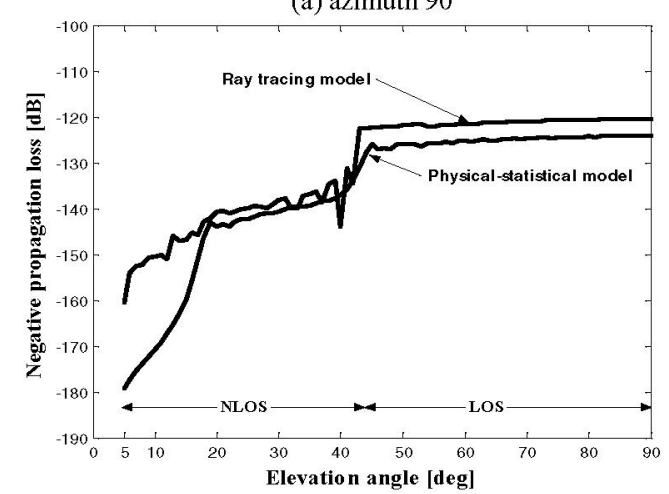

(b) azimuth $60^{\circ}$

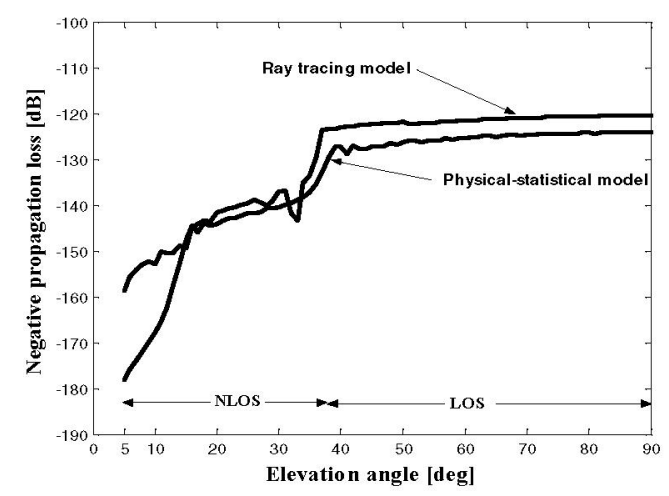

(c) azimuth $45^{\circ}$

Fig. 7 Predicted path loss for the model under test

Figs. 5 and 6 by making the scale to the SPF geometry.

The ray tracing simulation result is presented as a total propagation loss obtained from the calculation of direct LOS ray and multipath scattered rays of received electric field by vector summation using (10)-(12). Figs. 7 (a), (b), and (c) show the numerical result of propagation path loss prediction in different azimuth angles, those are $90^{\circ}, 60^{\circ}$, and $45^{\circ}$, respectively. In LOS region, path loss curve is obtained from direct and single ground or wall reflected ray. However, in NLOS region the rays come from reflected, rooftop diffracted and combination of diffracted-reflected rays. Note there is no direct ray in a NLOS region. Azimuth of $90^{\circ}$ demonstrates to be the worst scenario of propagation path loss due to building blockage. When MS in the shadow region associated to some particular elevation angles, the diffraction from rooftop is a major contributor to the received electric field. As a result, the 
propagation path loss dramatically increases to the value of the order of $20 \mathrm{~dB}$ or more compared with the LOS region. Considering the SPF coverage illustrated by (5), an average propagation path loss value is displayed in Table 3 for different azimuth angles. In Table 3, the propagation path loss was presented in an average value for the three regions within the coverage. Region 1 is a coverage with elevation angle greater than $45^{\circ}$ (within $20 \mathrm{~km}$ horizontal distance from sub-platform point). Region 2 is an area within elevation angle between $45^{\circ}$ and $15^{\circ}$ (correspond to horizontal distance between $20 \mathrm{~km}$ up to $75 \mathrm{~km}$ ), and region 3 is the rest of the coverage (from $75 \mathrm{~km}$ up to $194 \mathrm{~km}$ in horizontal distance).

TABLE III

Average Pathloss [dB] vs Elevation Angle for a Model in Fig. 5

\begin{tabular}{|c|c|c|c|}
\hline \multirow{2}{*}{ Azimuth [deg] } & \multicolumn{3}{|c|}{ Elevation angle [deg] } \\
\cline { 2 - 4 } & $\begin{array}{c}\text { Region 1 } \\
\geq 45\end{array}$ & $\begin{array}{c}\text { Region 2 } \\
45>\alpha \geq 15\end{array}$ & $\begin{array}{c}\text { Region 3 } \\
15>\alpha \geq 5\end{array}$ \\
\hline 90 & 121.6 & 142.3 & 172.8 \\
\hline 60 & 121.1 & 138.3 & 152.6 \\
\hline 45 & 121.2 & 135.6 & 151.4 \\
\hline
\end{tabular}

From the results we observed that azimuth angles have a little effect in high elevation angle such as in region 1. However azimuth angles have much an effect to the propagation loss in low elevation angle such as in region 2 and region 3. We observed that propagation loss in region 2 and 3 is decreasing as azimuth angle also decreases.

The results also show a very good agreement between ray tracing model and physical-statistical model for the scenario of $\theta=90^{\circ}$ in all elevation angles. In LOS condition, we observed the propagation loss obtained by using ray tracing model is about $3 \mathrm{~dB}$ lower than that by the physical-statistical model in all scenarios. This is due to we include the reflection from the ground and may be reflection from the building as well. In the NLOS condition, we noticed the results of both models are of little different. For both scenarios of $\theta=60^{\circ}$ and $\theta=45^{\circ}$, our ray tracing model exhibit higher received power level for low elevation angle (i.e. $\alpha<20^{\circ}$ ), while a very good agreement of both models can be observed for elevation angle higher than $20^{\circ}$. This is due to in our ray tracing model the contribution of the reflection and the diffraction rays and their combination are considered. However the physical-statistical model has considered only diffraction from rooftop of the building.

This propagation loss prediction actually depends on the model under consideration. These results could give much contribution to the analysis of co-channel interference experienced by the MS (downlink interference) or the SPF (uplink interference). This because of the propagation path losses are predicted more realistic compare to only assuming as a free-space path loss. For example, we will be able to calculate co-channel interference experienced by the MS that is receiving signal from wanted platform (say located at $80^{\circ}$ in elevation) but also suffer from unwanted platform signal (say located at $20^{\circ}$ in elevation). Whereas we found in a previous work the interference analysis for the case of IMT-2000 is based on a free space path loss assumption [11]-[13]. We will show the result of propagation path loss prediction to calculate required trans- mitted power at the SPF through the link budget analysis presented in the following section.

\section{LINK BUDGET ANALYSIS}

This section presents link budget analysis for the next generation of mobile communication digital CDMA-based such as IMT-2000. The required bit energy per noise power spectral density can be expressed as [19],

$$
\frac{E_{b}}{N_{0}}=\frac{P_{T} G_{t} G_{r}}{R_{b} k T_{0} L(\alpha, \theta) L_{0} M_{L}},
$$

where $R_{b}$ is the information rate in bits per second, $T_{0}$ is receiver temperature in degrees Kelvin, $k$ is Boltzmann's constant, $L_{\theta}$ is cable, connector, and combiner losses, $M_{L}$ is the link margin, and $L(\alpha, \theta)$ is a propagation path loss as a function of elevation and azimuth angle obtained in Section 5. In this analysis we evaluate the required transmitted power for different classes of services of the $3^{\text {rd }}$ generation multimedia application [20]. The parameters involved in the calculation are displayed on Table 4.

TABLE IV

ImT-2000 Specifications and SYstem Parameters Used In THE CALCulation

\begin{tabular}{|l|l|}
\hline \multicolumn{1}{|c|}{ Item } & Specification \\
\hline Frequency $[\mathrm{GHz}]$ & 2 \\
\hline Information rate $[\mathrm{kbps}]$ & $8,12.2,16,64,144,384,2000$ \\
\hline SPF antenna gain $[\mathrm{dBi}]$ & 30 \\
\hline MS antenna gain $[\mathrm{dBi}]$ & 0 \\
\hline Boltzmann's constant $[\mathrm{J} / \mathrm{K}]$ & $1.38 \times 10^{-23}$ \\
\hline Temperature's chamber $[\mathrm{K}]$ & 290 \\
\hline Link margin $[\mathrm{dB}]$ & 15.4 \\
\hline Cable, connector, \& other losses $[\mathrm{dB}]$ & 2 \\
\hline $\mathrm{E}_{\mathrm{b}} / \mathrm{N}_{0}[\mathrm{~dB}]$ & Max. 7.9 \\
\hline
\end{tabular}

The results are presented in Figs. 8 (a)-(c) for different scenarios, which means different azimuth angles those are $90^{\circ}$, $60^{\circ}$, and $45^{\circ}$, respectively. We observed in Fig. 8 (a), the required transmitted power for different scenarios is almost similar. This means in region 1 the required transmitted power at SPF is not much affected by the azimuth angle. However in Figs. 8 (b) and (c) the required transmitted power is dependent to the azimuth angle. Region 3 shows the worst scenario particularly for the azimuth angle of $90^{\circ}$. In this case, the required transmitted power was too high and may unable to be implemented for the SPF mobile communication system. For that case, the required transmitted power would have to vary between $31.2 \mathrm{~W}(8 \mathrm{kbps})$ up to $7.80 \mathrm{~kW}(2 \mathrm{Mbps})$.

\section{CONCLUSIONS}

This paper has demonstrated the ray tracing tool to examine the propagation path loss for a model of urban site mobile communication based on stratospheric platform. We developed the building block model, in which its geometry is based on the field survey of typical urban environment for evaluation. Simulated propagation path loss experienced by this system has been evaluated for various angles in terms of elevation and 
azimuth of the stratospheric platform as seen by the user. We have also shown the prediction result obtained by the physicalstatistical model for comparison with our result. A very good agreement can be observed especially for the scenario of $90^{\circ}$ azimuth angle. Furthermore, the required transmitted power by the stratospheric platform has been examined through the link budget analysis at the standard transmission rates for the application of IMT-2000. The critical required transmitted power in the model is observed for region 3 that the azimuth angle is $90^{\circ}$. Such highly required transmitted power may not be able to be implemented in mobile communication employing the concept of the stratospheric platform.

\section{REFERENCES}

[1] G.M. Djuknic, J. Freidenfelds, and Y. Okunev, "Establishing wireless communications services via high altitude aeronautical platforms: A concept whose time has come?," IEEE Commun. Mag., vol. 35, no. 9, pp. 128-135, Sept. 1997.

[2] Y. Hase, R. Miura, and S. Ohmori, "A novel broadband all-wireless access network using stratospheric platforms," Proc. $48^{\text {th }}$ IEEE ITC Spring, vol. 2, May 1998, pp. 1191-1194.

[3] T.C. Tozer and D. Grace, "High-altitude platforms for wireless communications," IEE Elec. and Commun. Eng. J., vol. 13, no. 3, pp. 127-137, June 2001.

[4] R. Miura and M. Oodo, "Wireless communications system using stratospheric platforms," J. Commun. Research Lab., vol. 48, no. 4, pp. $33-48,2001$.

[5] "Minimum performance characteristics and operational conditions for high altitude platform stations providing IMT-2000 in the bands 1885$1980 \mathrm{MHz}, 2010-2025 \mathrm{MHz}$, and $2110-2170 \mathrm{MHz}$ in the Regs. 1 \& 3 and $1885-1980 \mathrm{MHz}$ and $2110-2160 \mathrm{MHz}$ in Reg. 2," ITU-R M. 1456 , 2000 .

[6] "Preferred characteristics of systems in the fixed service using highaltitude platform stations operating in the bands $47.2-47.5 \mathrm{GHz}$ and 47.9-48.2 GHz," Recommendation ITU-R F.1500, 2000.

[7] M. Oodo, R. Miura, T. Hori, T. Morisaki, K. Kashiki, M. Suzuki, "Sharing and compatibility study between fixed service using high altitude platform stations (HAPS) and other services in the $31 / 28 \mathrm{GHz}$ bands," Wireless Personal Commun., vol. 23, pp. 3-14, 2002.

[8] D. Grace, J. Thornton, G. Chen, G.P. White, T.C. Tozer, "Improving the system capacity of broadband services using multiple high-altitude platforms," IEEE Trans. on Wireless Commun., vol. 4, no. 2, pp. 700709, March 2005

[9] B. El-Jabu and R. Steele, "Cellular communication using aerial platform," IEEE Trans. on Vehicular Technology, vol. 50, no. 3, pp. 668-700, May 2001

[10] J. Thornton, D. Grace, M.H. Capstick, T.C. Tozer, "Optimizing an array of antennas for cellular coverage from a high altitude platform," IEEF Trans. on Wireless Commun., vol. 2, issue 3, pp. 484-492, May 2003.

[11] Jong-Min Park, Dae-Sub Oh, Yang-Sun Kim, and Do-Seob Ahn, "Evaluation of interference effect into cellular system from high altitude platform station to provide IMT-2000 service," Proc. IEEE Globecom '03, vol. 1 , Dec. 2003, pp. 420-424.

[12] S. Masumura and M. Nakagawa, "Joint system of terrestrial and high altitude platform station (HAPS) cellular for W-CDMA mobile communication," IEICE Trans. Commun., vol. E. 85, no. 10, pp. 20512058 , Oct. 2002.

[13] E. Falletti, M. Mondin, F. Dovis, and D. Grace, "Integration of a HAP within a terrestrial UMTS network: interference analysis and cell dimensioning," Wireless Personal Commun., vol. 24, pp. 291-325, 2003.

[14] S. Shimamoto, T. Mikoshiba, S. Takakusagi, M. Hayashi, and H. Shiba, "Performance evaluations of communication systems employing stratospheric aircrafts and LEO satellites," IEICE Trans. Commun., vol. E81-B, no. 12, pp. 2343-2350, Dec. 1998

[15] Iskandar and S. Shimamoto, "Channel characterization and performance evaluation of mobile communication employing stratospheric platforms," IEICE Trans. Commun., vol. E89-B, no. 3, March 2006.

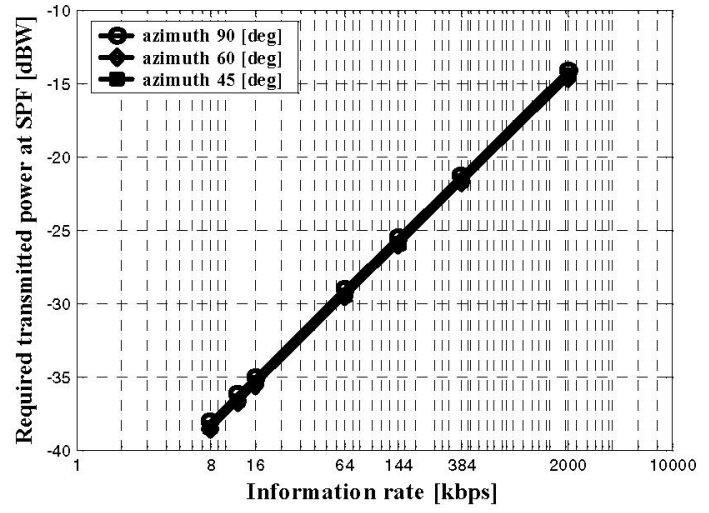

(a) Region 1

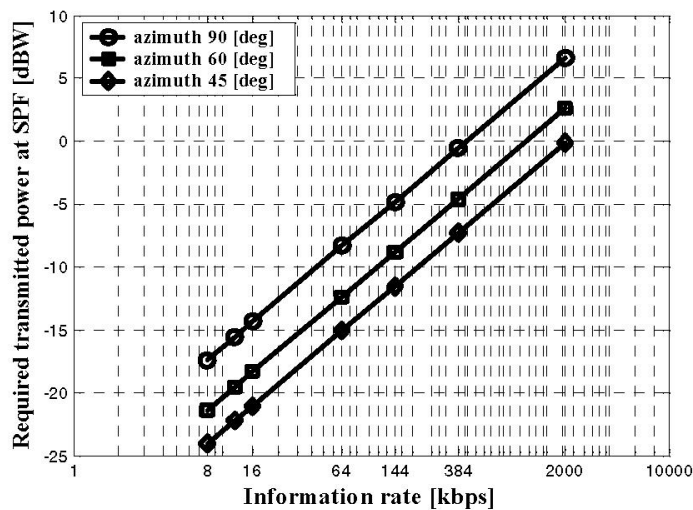

(b) Region 2

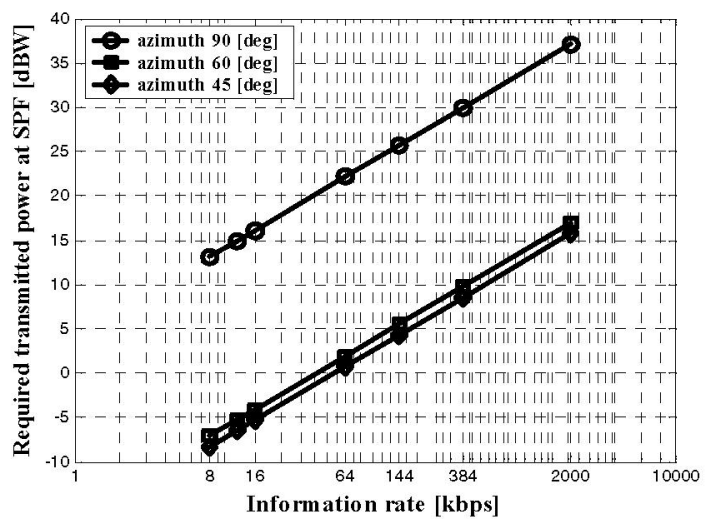

(c) Region 3

Fig. 8 Required transmitted power at the SPF

[16] F. Tila, P.R. Shepherd and S.R. Pennock, "Analysis of indoor-indoor and satellite/HAP-indoor propagation effects," Proc. $11^{\text {th }}$ Int. Conf. on Antennas and Propagation, vol. 1, April 2001, pp. 203-207.

[17] H.L. Bertoni, Radio Propagation for Modern Wireless Systems. Prentice-Hall PTR, 2000.

[18] S.R. Saunders and B.G. Evans, "A physical statistical model for land mobile satellite propagation in built-up areas," Proc. $10^{\text {th }}$ Int. Conf. on Antennas and Propagation, vol. 2, April 1997, pp. 44-47.

[19] John G. Proakis, Digital Communications. Mc Graw Hill, 2000.

[20] H. Holma and A. Toskala, W-CDMA for UMTS: Radio Access for Third Generation Communication. John Wiley and Sons, 2000. 